\title{
EPIDEMIOLOGY
}

\section{Periodicity of dengue}

New research modelling the epidemiology of dengue haemorrhagic fever (DHF) in Thailand has uncovered a 3-year periodicity in the magnitude of disease incidence, and a travelling wave of infection that spreads outwards from urban centres.

Of the 50-100 million people each year that are infected with mosquito-borne Dengue virus, up to 500,000 develop the potentially fatal DHF. Because the incidence of DHF can fluctuate tenfold from one year to the next, epidemics can overwhelm healthcare providers.

Using the statistical tool empirical mode decomposition (EMD) to analyse the monthly incidence of DHF in 72 provinces of Thailand over a 14-year period, the authors found a 3-year periodicity of outbreaks that was masked in the raw data. This periodicity is reminiscent of host-pathogen and predator-prey relationships, but hasn't been observed before for a vector-borne disease.
By correlating the periodicity with the distance of provinces from Bangkok, a clear pattern emerged -68 provinces had a pattern that was synchronized with, but lagged behind, Bangkok. DHF emanated out from Bangkok at $148 \mathrm{~km}$ per month as a travelling wave, which had a broad range affecting almost the whole country. Border regions conflicted with the pattern and the authors speculate that other urban areas might affect spread as well.

Why does a wave emanate from Bangkok? One reason might be complex interactions between the four different serotypes of dengue virus and a large urban host population.

There are few tools other than vector control to prevent dengue virus transmission, so this model could be valuable in reducing the burden of this disease.

Susan Jones

\section{9) References and links}

ORIGINAL RESEARCH PAPER Cummings, D. A. T. et al.

Travelling waves in the occurrence of dengue haemorrhagic fever in Thailand. Nature 427, 344-347 (2004)

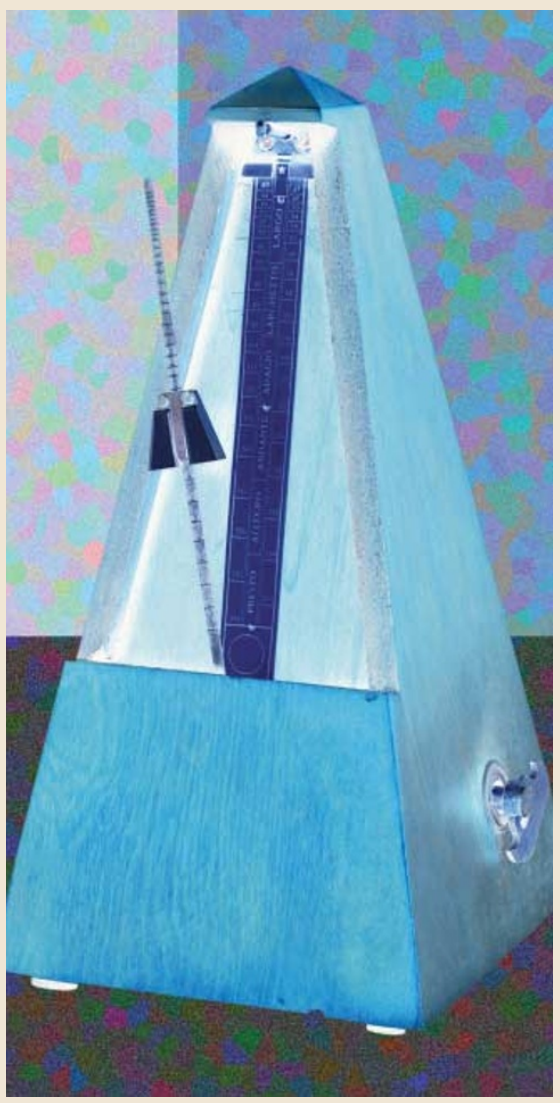

\section{ANTI-INFECTIVES}

\section{A salutary lesson}

A recent report in Antimicrobial Agents and Chemotherapy has shown that, for one promising antimicrobial peptide, in vitro measures of bacteriocidal efficacy cannot be used to predict in vivo performance. Although novispirin G10 is significantly less toxic than its parent compounds and retains high antimicrobial activity against bacteria, when used in a mouse model of lung infection, the toxicity of the molecule is extensive.

In recent years, an area of focus in the ongoing search for new anti-infective agents

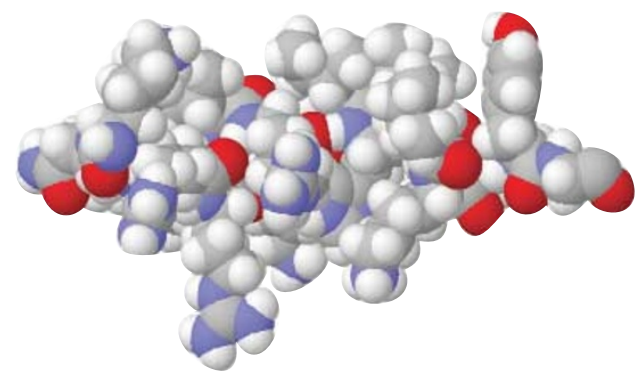

Three-dimensional solution structure of novispirin G10. PDB ID: 1HU6. Sawai, M.V. et al. Protein Eng. 15, 225-232 (2002) has centred on peptides that function in the innate immune systems of plants and animals. One class of promising antimicrobial peptides are the mammalian-derived cathelicidins isolated from leukocytes and epithelial cells. Although earlier work showed that the parent molecule exhibited high cytotoxic activity in human cells, synthetic analogues offered the promise of potent, broad-based antimicrobial efficacy. To investigate the properties of one such analogue, novispirin G10, both alone and in combination with an established pulmonary therapeutic agent (tobramycin), Peter Thorne and colleagues developed a mouse model of sub-acute bacterial lung infection. The authors challenged the mice with Klebsiella pneumoniae, an organism known to elicit a strong cytokine response in mouse lung and which can resist clearance for 48-72 hours, thereby approximating a chronic infection. It was hypothesized that the peptide would bind bacterial lipopolysaccharide, reducing the endotoxic potential for lung damage.

However, contrary to what was predicted, the inclusion of novispirin G10 in the treatment regimen increased both the inflammatory and toxic responses of the host. Furthermore, the authors were able to recover significantly more bacteria from mice that were administered the peptide, an observation that correlated with strong evidence of inflammation and lung toxicity. IL- 6 and TNF- $\alpha$ are proinflammatory cytokines that are sensitive to endotoxin stimulation and, if upregulated, they contribute to pulmonary disease in Gram-negative bacterial infections. When the authors measured the levels of circulating IL-6 in mice infected with K. pneumoniae, the concentration of cytokine was 1,000-10,000-fold higher than in noninfected controls, with the highest levels in mice treated with novispirin G10 alone, or in combination with tobramycin. Their studies demonstrated that novispirin G10 was relatively non-toxic in control mice but was highly toxic in mice infected with K. pneumoniae.

The mechanisms for the surprising effects observed in this study are unknown and are the subject of further study. What is clear, however, is that these findings re-enforce the crucial importance of investigating candidate anti-infective agents as soon as possible in a relevant in vivo animal model.

David O'Connell

(2) References and links

ORIGINAL RESEARCH PAPER Bartlett K. H. et al. Novispirin G10-induced lung toxicity in a Klebsiella pneumoniae infection model. Antimicrob. Agents Chemother. 47, 3901-3906 (2003) FURTHER READING Ganz, T. Antimicrobial polypeptides in host defence of the respiratory tract. J. Clin. Invest. 109, 693-697 (2002)

\section{WEB SITE}

Environmental Health Sciences Research Center, University of lowa: http://www.ehsrc.org 